\title{
Quiet-time low energy ion spectra observed on Solar Orbiter during solar minimum
}

G. M. Mason ${ }^{1} \odot$, G. C. $\mathrm{Ho}^{1}$, R. C. Allen ${ }^{1}$, Z. G. Xu ${ }^{2}$, N. P. Janitzek ${ }^{3}$, J. L. Freiherr von Forstner ${ }^{2}$, A. Kohllhoff ${ }^{2}$, D. Pacheco ${ }^{2}$, J. Rodríguez-Pacheco ${ }^{4}$, R. F. Wimmer-Schweingruber ${ }^{2}$, G. Bruce Andrews ${ }^{1}$, C. E. Schlemm ${ }^{1}$, H. Seifert ${ }^{1}$, K. Tyagi ${ }^{1,5}$, W. J. Lees ${ }^{1}$, J. Hayes ${ }^{1}$, R. Gómez-Herrero ${ }^{4}$, M. Prieto ${ }^{4}$, S. Sánchez-Prieto ${ }^{4}$, F. Espinosa Lara ${ }^{4}$, I. Cernuda ${ }^{4}$, P. Parra Espada ${ }^{2}$, O. Rodríguez Polo ${ }^{4}$, A. Martínez Hellín², C. Martin²,6, S. Böttcher², L. Berger ${ }^{2}$, J. C. Terasa ${ }^{2}$, S. Boden ${ }^{2,7}$, S. R. Kulkarni ${ }^{2,8}$, A. Ravanbakhsh ${ }^{2,9}$, M. Yedla ${ }^{2,9}$, S. Eldrum ${ }^{2}$, R. Elftmann ${ }^{2}$, and P. Kühl ${ }^{2}$

\footnotetext{
1 Johns Hopkins Univ. Applied Physics Laboratory, Laurel, MD, USA e-mail: glenn.mason@jhuapl.edu

2 Institut für Experimentelle und Angewandte Physik, Christian-Albrechts-Universität zu Kiel, Kiel, Germany

3 ESA-ESAC, Madrid, Spain European Space Agency (ESA/ESAC), Villafranca del Castillo, 28692 Madrid, Spain

${ }^{4}$ Space Research Group, Universidad de Alcalá, Alcalá de Henares, Spain

5 Now at Univ. Colorado/LASP, Boulder, CO, USA

${ }^{6}$ Now at: German Aerospace Center (DLR), Dept. of Extrasolar Planets and Atmospheres, Berlin, Germany

7 Now at: DSI Datensicherheit GmbH, Rodendamm 34, 28816 Stuhr, Germany

8 Now at: Deutsches Elektronen-Synchrotron (DESY), Platanenallee 6, 15738 Zeuthen, Germany

9 Now at: Max-Planck-Institute for Solar System Research, Justus-von-Liebig-Weg 3, 37077 Göttingen, Germany
}

Received 11 February 2021 / Accepted 16 March 2021

\begin{abstract}
Context. The Solar Orbiter spacecraft cruised in the inner heliosphere during Feb. 2020 - Jan. 2021, moving between $\sim 0.5-1.0$ au radial distance. The Energetic Particle Detector suite operated continuously during this period.

Aims. The Suprathermal Ion Spectrograph and High Energy Telescope observations made during intervals in between transient intensity increases were used to determine the low energy ion spectra and composition during quiet times.

Methods. Energetic particle spectra and major ion components, including ${ }^{3} \mathrm{He}$, were measured over the range $\sim 0.1-$ $100 \mathrm{MeV}$ nucleon ${ }^{-1}$. The radial dependence of $4.4 \mathrm{MeV}$ nucleon $^{-1}{ }^{4} \mathrm{He}$ and $\mathrm{O}$ was measured. A short interval of extremely low intensities ("super-quiet") was also studied.

Results. Spectra measured during the quiet period showed transitions, including galactic cosmic rays $\left(>50 \mathrm{MeV}\right.$ nucleon ${ }^{-1}$ ), anomalous cosmic rays (a few to $\sim 50 \mathrm{MeV}$ nucleon $^{-1}$ ), and a steeply rising "turn-up" spectrum below a few $\mathrm{MeV}$ nucleon ${ }^{-1}$ whose composition resembled impulsive, ${ }^{3} \mathrm{He}$-rich solar energetic particle events. The radial dependence had large uncertainties but was consistent with a small gradient. During the super-quiet interval, the higher energy components remained similar to the quiet period, while the approximately flat low energy ${ }^{4} \mathrm{He}$ spectrum extended downward, reaching $\sim 300 \mathrm{keV}$ nucleon ${ }^{-1}$ before transitioning to a steeply rising spectrum.
\end{abstract}

Key words. acceleration of particles - Sun: abundances - Sun: particle emission - interplanetary medium - cosmic rays

\section{Introduction}

During so-called quiet periods when transient solar particle events, interplanetary shocks, and other activity is absent, low energy ion spectra in the interplanetary medium near 1 au are formed of three components, as illustrated in Fig. 1: From high energy to low energy, these are galactic cosmic rays (GCRs), anomalous cosmic rays (ACRs), and a "turn-up" spectrum below a few $\mathrm{MeV}$ nucleon $^{-1}$ that increases with decreasing energy. While the GCRs and ACRs vary with solar cycle timescales, the turn-up spectrum is variable on shorter timesscales and may be dominated by remnant solar energetic particles, stream interaction, and shock-accelerated particles, as well as more local sources such as comets or planetary upstream magnetospheric events (e.g., overviews by Klecker et al. 1977; Reames 1999; Desai et al. 2008; Dayeh et al. 2009; Mason \& Gloeckler 2011).

Anomalous cosmic rays originate from interstellar neutrals entering the outer heliosphere, followed by ionization and acceleration that is presumably in association with the termination shock, and penetrate into the inner heliosphere where their intensity is reduced by solar modulation (e.g., Klecker 1999; Cummings et al. 2002; McComas \& Schwadron 2006). Although well studied at 1 au and beyond, there have been very few observations of ACRs inside 1 au. Solar Orbiter offers the opportunity to study this component in detail inside Earth's orbit.

The variable turn-up spectrum at low energies has been investigated to ascertain whether these particles have a solar origin or are modulated GCRs (Krimigis et al. 1973; Zamow 1975), 


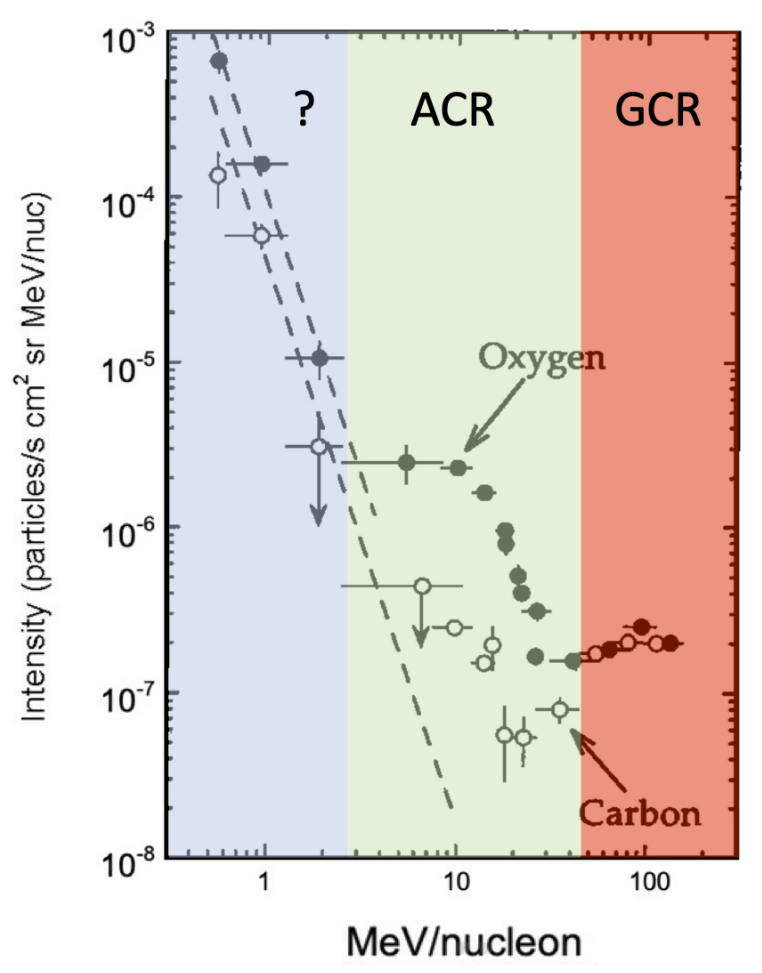

Fig. 1. Quiet-time energy spectra observed near solar minimum at $1.0 \mathrm{au}$ on the Solar, Anomalous, and Magnetospheric Particle Explorer (SAMPEX) satellite in 1992-1993; colored shading approximately separates three energy regimes and their associated spectra. Left: low energy "turn-up" spectra of undetermined origin. Center: anomalous cosmic ray $\mathrm{O}$ and C. Right: modulated GCRs. The figure is adapted from Mewaldt et al. (1993).

accelerated solar wind, or some other steady emission from the Sun (Fisk \& Gloeckler 2006; Mason \& Gloeckler 2011). Characterizing this population is important for determining the characteristics of the inner heliosphere suprathermal ion pool, which provides an important seed population for energetic particles energized by interplanetary shocks (Kahler \& Ling 2019). Solar Orbiter has the potential to distinguish between different models for this component by determining, for example, whether it is more or less intense closer to the Sun or at latitudes off the ecliptic plane. In this Letter we present the first observations from Solar Orbiter that address these questions.

\section{Observations}

The particle observations reported here were made on the Solar Orbiter spacecraft (Müller et al. 2020) with the Suprathermal Ion Spectrograph (SIS) and High Energy Telescope (HET) which are part of the Energetic Particle Detector (EPD) suite (Rodríguez-Pacheco et al. 2020). The SIS is a time-of-flight mass spectrometer that measures ion composition from $\sim 0.1-$ $10 \mathrm{MeV}$ nucleon $^{-1}$, and HET is a $\mathrm{d} E / \mathrm{d} x$ versus $E$ telescope that measures particle composition from $\sim 10$ to several hundred $\mathrm{MeV}_{\text {nucleon }}{ }^{-1}$. For the SIS observations, the background was suppressed by requiring consistency between the instrument's two independent mass measurements.

\subsection{Selection of time intervals}

The definition of quiet time at energies below a few $\mathrm{MeV}_{\text {nucleon }}{ }^{-1}$ is subjective because there is no identifiable

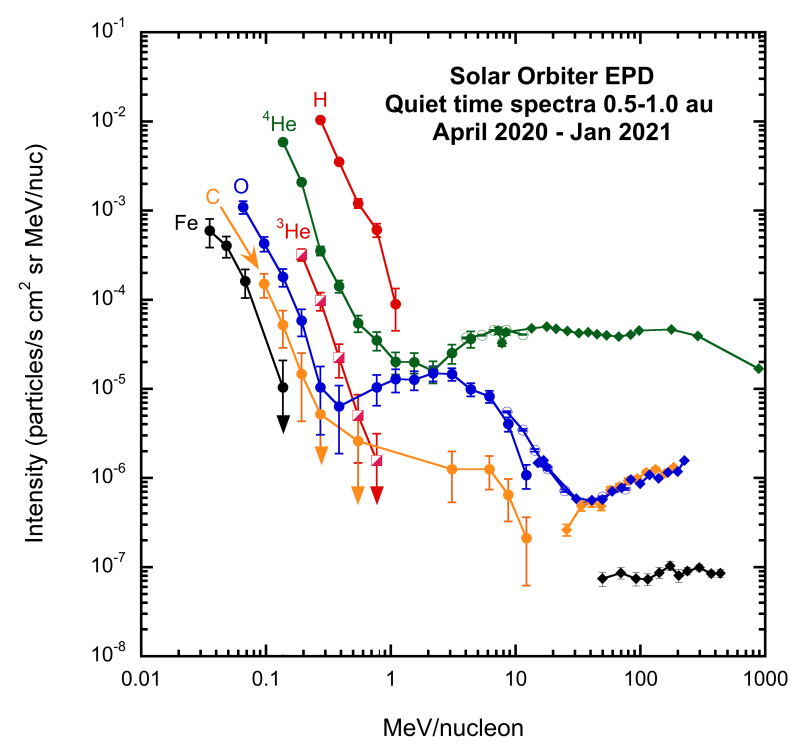

Fig. 2. Quiet-time spectra observed on Solar Orbiter between 0.5 and 1.0 au by EPD/SIS (filled circles and half-filled squares), EPD/HET (filled diamonds), and ACE at 1 au (open circles). Downward pointing arrows signify one-count upper limits.

minimum count rate at these energies, rather a continuous range of intensity values whose lower limit depends on many factors, such as instrument energy threshold, background and/or sensitivity (e.g., Dayeh et al. 2009; Richardson et al. 1990), as well as activity levels in interplanetary space. This Letter analyzed two intervals: first, a "quiet" interval where obvious increases in the hourly averaged $273 \mathrm{keV}$ nucleon ${ }^{-1} \mathrm{H}$ and He intensities showed the presence of transient events that were removed from the analysis (see Allen et al. 2021; Mason et al. 2021). The quiet period has 2386 hours of collection time from April 2020 through January 2021 and is used for the observations in Figs. 2 and A.1.

Second, we selected a "super-quiet" interval, the quietest multiday interval during the study, in order to measure the residual spectra at the lowest levels below $1 \mathrm{MeV}$ nucleon $^{-1}$ (e.g., Mason et al. 1979; Mason \& Gloeckler 2011). Appendix B describes the identification of this 192-hour super-quiet interval, which is used for Fig. 3.

\subsection{Anomalous cosmic rays and low energy turn-up in the quiet-time interval}

Figure 2 shows the quiet-time EPD spectra, with small coverage gaps in $\mathrm{He}$ and $\mathrm{O}$ covered by quiet data from the Advanced Composition Explorer (ACE) Solar Isotope Spectrometer. The ACR $\mathrm{O}$ in Fig. 2 rises from the GCR spectrum at $\sim 30 \mathrm{MeV}$ nucleon $^{-1}$, similar to the 1992-1993 spectra in Fig. 1. However, the 1992-1993 ACR spectrum flattens around $10 \mathrm{MeV}$ nucleon $^{-1}$, although the wide energy bin from $2.5-10 \mathrm{MeV}$ nucleon $^{-1}$ might conceal more detail. In any case, the 2020-2021 ACR O keeps rising, with a peak near $4 \mathrm{MeV}$ nucleon $^{-1}$ that is about five times higher than the Fig. 1 spectrum; this spectrum was measured somewhat before solar minimum but reached levels near that shown in Fig. 2 by 1994 (Klecker et al. 1998). The ACR O in Fig. 2 decreases from this local maximum then transitions to the turn-up at about $300 \mathrm{keV}$ nucleon $^{-1}$, about a factor of ten lower energy than the start of the turn-up in the 1992-1993 data. In the range $\sim 10$ $100 \mathrm{MeV}$ nucleon ${ }^{-1}$, the ${ }^{4} \mathrm{He}$ intensity of $\sim 4 \times 10^{-5}\left(\mathrm{~s}^{-1} \mathrm{~cm}^{2} \mathrm{sr}\right.$ $\mathrm{MeV}$ nucleon $^{-1}$ ) is similar to previously measured spectra near 


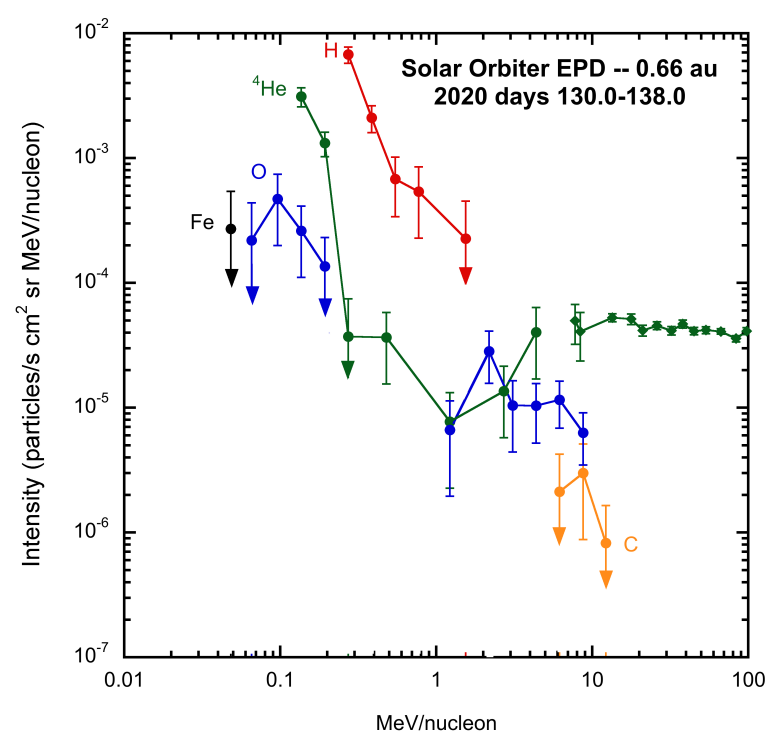

Fig. 3. Low energy spectra during the super-quiet period; symbols are the same as in Fig. 2.

solar minimum (Garcia-Munoz et al. 1977; Reames et al. 1997). The radial gradient in ${ }^{4} \mathrm{He}$ and $\mathrm{O}$ near the peak of the spectrum is discussed in Appendix A.

\subsection{Low energy spectra in a super-quiet period}

In order to further explore the turn-up spectra, we studied a superquiet interval selected as described in Appendix B. Figure 3 shows the super-quiet period particle spectra. In the low energy turn-up portion the intensities are about half that shown in the period in Fig. 2. Above $1 \mathrm{MeV}$ nucleon $^{-1}$, the $\mathrm{He}$ and $\mathrm{O}$ flatten due to ACRs. Although ${ }^{3} \mathrm{He}$ was present in Fig. 2, it is absent here. Examining the spectral shapes in Fig. 3, the ${ }^{4} \mathrm{He}$ spectrum is consistent with the higher energy ACRs down to about $300 \mathrm{keV}$ nucleon ${ }^{-1}$, although the statistical uncertainties are large. Below $300 \mathrm{keV}$ nucleon ${ }^{-1}$, there is a large increase in intensity for both ${ }^{4} \mathrm{He}$ and $\mathrm{O}$. Although the data in Fig. 3 are at the limits of the instrument sensitivity, they are consistent with an ACR ${ }^{4} \mathrm{He}$ source down to $300 \mathrm{keV}$ nucleon ${ }^{-1}$. However, the errors are large enough that other possibilities cannot be ruled out.

\section{Discussion and conclusions}

The ACR spectra shown in Fig. 2 are similar to observations in prior solar cycles. However, they also show the ACR O spectrum with a local maximum at $\sim 2-4 \mathrm{MeV}$ nucleon $^{-1}$, rather than a plateau, and a small decrease below $\sim 4 \mathrm{MeV}$ nucleon $^{-1}$. Since most prior ACR measurements start at several $\mathrm{MeV}$ nucleon $^{-1}$ this feature cannot be compared with the earlier work. In the range 8-27 MeV nucleon ${ }^{-1}$ Mewaldt et al. (1993) reported very similar maximum ACR O intensities in the 1972, 1987, and 1993 solar minima, where in each case the intensity decreased by an order of magnitude when modulation reached solar maximum values. They pointed out that this closely repeatable level at solar minimum is also mirrored in much higher energy neutron monitor data. Jokipii et al. (1977) showed that ACRs enter the heliosphere either drifting along the neutral sheet, or flowing down the solar polar regions to the equatorial region, depending on the 22-year polarity of the solar cycle. The current solar minimum corresponds to the $A>0$ drift condition, where ACRs drift in from the polar regions and, therefore, a positive latitudinal gradient is predicted, which has been observed beyond 1 au (Cummings et al. 1995; McKibben 1989). As Solar Orbiter reaches higher latitudes later in the mission the polarity of the current cycle predicts a positive gradient, although close to the Sun the effect may be small.

We now consider the turn-up spectra, first for the quiet time. The spectra and composition, especially with the high ${ }^{3} \mathrm{He} /{ }^{4} \mathrm{He}$ ratio, are consistent with impulsive solar energetic particle (SEP) material. Previously, Richardson et al. (1990), Desai et al. (2006), and Dayeh et al. (2009) found high ${ }^{3} \mathrm{He} /{ }^{4} \mathrm{He}$ in quiet periods during solar maximum, with the ${ }^{3} \mathrm{He}$ nearly disappearing during solar minimum. The present solar minimum quiet time has ${ }^{3} \mathrm{He} /{ }^{4} \mathrm{He} \sim 20 \%$ below $1 \mathrm{MeV}$ nucleon ${ }^{-1}$, considerably higher than the previous results. This might be due to a combination of the observations being closer to the Sun, where the impulsive SEP intensities are higher and a lower general level of corotating interaction region (CIR) intensities, which fall off strongly inside 1 au (Allen et al. 2021). Where comparisons can be made, the overall intensity levels in Fig. 2 are generally similar to the earlier surveys. Below $1 \mathrm{MeV}$ nucleon ${ }^{-1}$, the $\mathrm{H}$ and ${ }^{4} \mathrm{He}$ power-law spectral slopes are -2.8 and -3.1 , respectively, and are similar to the $\mathrm{H}$ and $\mathrm{He}$ spectral slopes $<10 \mathrm{MeV}$ nucleon $^{-1}$ summarized by Richardson et al. (1990).

Considering the super-quiet period, the $\mathrm{H}$ and He turn-up spectra in Fig. 3 are about one-half the intensity of the previously reported super-quiet periods in 1977 and 2008-2009 (Mason \& Gloeckler 2011). Given the large variability of intensities observed at low energies, it is surprising that the present $\mathrm{H}$ and ${ }^{4} \mathrm{He}$ intensities are so similar to those reported earlier. For $\mathrm{H}$ and ${ }^{4} \mathrm{He}$, the measurements are statistically accurate, so for these species instrument sensitivity is not a limiting factor. The proton spectral slope is -2.5 .

A basic question is whether when SEPs and other transients are removed, there remains a continuous source of energetic ions in the interplanetary medium, for example, from either heated solar material or cooled GCRs. The presence or lack of such energized ions can provide insights into possibly new acceleration processes in the interplanetary medium or solar corona. In the super-quiet period at $0.66 \mathrm{au}$, between $300 \mathrm{keV}$ nucleon $^{-1}$ and a few MeV nucleon ${ }^{-1}$, the ${ }^{4} \mathrm{He}$ is consistent with an extension of the ACR spectrum to lower energies, but the lack of observations of heavier elements in this range leaves the source properties ambiguous. However, we find the turn-up spectra still present, with protons extending to $\sim 1 \mathrm{MeV}$, but with ${ }^{4} \mathrm{He}$ showing a sharp drop-off above $200 \mathrm{keV}$ nucleon ${ }^{-1}$. The difference between the $\mathrm{H}$ and ${ }^{4} \mathrm{He}$ drop-off energies may be a clue for the accelerating mechanism. At even lower energies down to the solar wind peak, other sources, such as inner source material or continuously heated solar wind, may be critically important (e.g., Gloeckler \& Geiss 1998; Fisk \& Gloeckler 2006).

The super-quiet turn-up spectra reported here need to be examined in the context of multiple sources that contribute to the suprathermal ion pool within $1 \mathrm{au}$. Intensities as shown in Fig. B.1 make it clear that during solar minimum energetic particles associated with solar wind streams are the most common population to dominate intensities, followed by SEP events. During solar active periods this situation is reversed as SEPs become more common. The surveys of Desai et al. (2006) and Dayeh et al. (2009) showed, however, that impulsive ${ }^{3}$ He-rich SEP events dominate, apparently since their frequency of occurrence compensates for their smaller size compared to large gradual events (see also Wiedenbeck et al. 2005; Wang et al. 2012). 
Considering regions closer to the Sun than $0.5 \mathrm{au}$, where the bulk of energetic particles may be accelerated in largescale shock-associated events, the mix of quiet-time particles may change. The stream-associated events important inside 1 au show a large negative gradient (Allen et al. 2021, and references therein) and may therefore be much less important close to the Sun. Additionally, the impulsive ${ }^{3} \mathrm{He}$-rich material reported here in the quiet turn-up very possibly is not coming directly from active regions on the Sun, but rather is remnant material from earlier events that finds its way into the inner heliosphere: This can be tested in future Solar Orbiter observations as the perihelion decreases later in the mission and the solar activity cycle progresses.

Acknowledgements. The Suprathermal Ion Spectrograph (SIS) is a European facility instrument funded by ESA. The SIS instrument was constructed by the JHU/Applied Physics Lab. and CAU Kiel. We thank the many individuals at ESA and within the Energetic Particle Detector team for their support in its development. Post launch operation of SIS at APL is funded by NASA contract NNN06AA01C, and we thank NASA headquarters and the NASA/GSFC Solar Orbiter project office for their continuing support. The UAH team acknowledges the financial support by the Spanish Ministerio de Ciencia, Innovación y Universidades FEDER/MCIU/AEI Projects ESP2017-88436 $\mathrm{R}$ and PID2019-104863RB- I00/AEI/10.13039/501100011033. The CAU Kie team thanks the German Federal Ministry for Economic Affairs and Energy and the German Space Agency (Deutsches Zentrum für Luft- und Raumfahrt, e.V., (DLR)) for their unwavering support under grant numbers 50OT0901, 50OT1202, 50OT1702, and 50OT2002; and ESA for supporting the build of SIS under contract number SOL.ASTR.CON.00004, and the University of Kiel and the Land Schleswig-Holstein for their support of SIS. We acknowledge data from the ACE web site from the Caltech Solar Isotope Spectrometer instrument and SWICS solar wind instrument.

\section{References}

Allen, R. C., Mason, G. M., Ho, G. C., et al. 2021, A\&A, 656, L2 (SO Cruise Phase SI)
Cummings, A. C., Mewaldt, R. A., Cummings, J., et al. 1995, Geophys. Res. Lett., 22, 341

Cummings, A. C., Stone, E. C., \& Steenberg, C. D. 2002, ApJ, 578, 194

Dayeh, M. A., Desai, M. I., Dwyer, J. R., et al. 2009, ApJS, 693, 1588

Desai, M. I., Mason, G. M., Mazur, J. E., \& Dwyer, J. 2006, ApJ, 645, L81

Desai, M. I., Mason, G. M., Müller-Mellin, R., et al. 2008, J. Geophys. Res., 113, 8103

Fisk, L. A., \& Gloeckler, G. 2006, ApJ, 640, L79

Garcia-Munoz, M., Mason, G. M., \& Simpson, J. 1977, ApJ, 213, 263

Gloeckler, G., \& Geiss, J. 1998, Space Sci. Rev., 86, 127

Jokipii, J. R., Levy, E. H., \& Hubbard, W. B. 1977, ApJ, 213, 861

Kahler, S. W., \& Ling, A. G. 2019, ApJ, 872, 89

Klecker, B. 1999, Adv. Space Res., 23, 521

Klecker, B., Hovestadt, D., Gloeckler, G., \& Fan, C. Y. 1977, ApJ, 212, 290

Klecker, B., Mewaldt, R. A., Bieber, J. W., et al. 1998, Space Sci. Rev., 83 259

Krimigis, S. M., Armstrong, T. P., \& Kohl, J. W. 1973, ICRC, 13, 1656

Marquardt, J., Heber, B., Potgieter, M. S., \& Strauss, R. D. 2018, A\&A, 610, A42

Mason, G. M., \& Gloeckler, G. 2011, Space Sci. Rev., 98

Mason, G. M., Gloeckler, G., \& Hovestadt, D. 1979, in ICRC 16, 1, 259

Mason, G. M., Ho, G. C., Allen, R. C., et al. 2021, A\&A, 656, L1 (SO Cruise Phase SI)

McComas, D. J., \& Schwadron, N. A. 2006, Geophys. Res. Lett., 33, 4102

McKibben, R. B. 1989, J. Geophys. Res., 94, 17021

Mewaldt, R. A., Cummings, A. C., Cummings, J. R., et al. 1993, Geophys. Res. Lett., 20, 2263

Müller, D., Cyr, O. C. S., Zouganelis, I., Gilbert, H. E., \& Marsden, R. 2020 , A\&A, 642, A1

Rankin, J. S., McComas, D. J., Leske, R. A., et al. 2021, ApJ, 912, 139

Reames, D. V. 1999, ApJS, 518, 473

Reames, D. V., Barbier, L. M., \& von Rosenvinge, T. T. 1997, Adv. Space Res. 19, 809

Richardson, I. G., Reames, D. V., Wenzel, K., \& Rodriguez-Pacheco, J. 1990, ApJ, 363, L9

Rodríguez-Pacheco, J., Wimmer-Schweingruber, R. F., Mason, G. M., et al. 2020, A\&A, 642, A7

Wang, L., Lin, R. P., Krucker, S., \& Mason, G. M. 2012, ApJS, 759, 69

Wiedenbeck, M. E., Mason, G. M., Cohen, C. M. S., et al. 2005, in ICRC 29, 117

Zamow, R. 1975, ApJ, 197, 767 


\section{Appendix A: ACR radial gradient inside 1 au}

Figure A.1 shows the ${ }^{4} \mathrm{He}$ and $\mathrm{O}$ intensities near $4.4 \mathrm{MeV}$ nucleon $^{-1}$ for three radial distances. The combination of a low count rate along with small $\Delta r$ leads to large uncertainties, but it is consistent with the small positive $\mathrm{O}$ radial gradient observed on Helios and Parker Solar Probe (Marquardt et al. 2018; Rankin et al. 2021), as well as over larger distances beyond 1 au (Cummings et al. 1995).
He and $\mathrm{O}$ intensities 4.4 MeV/nucleon

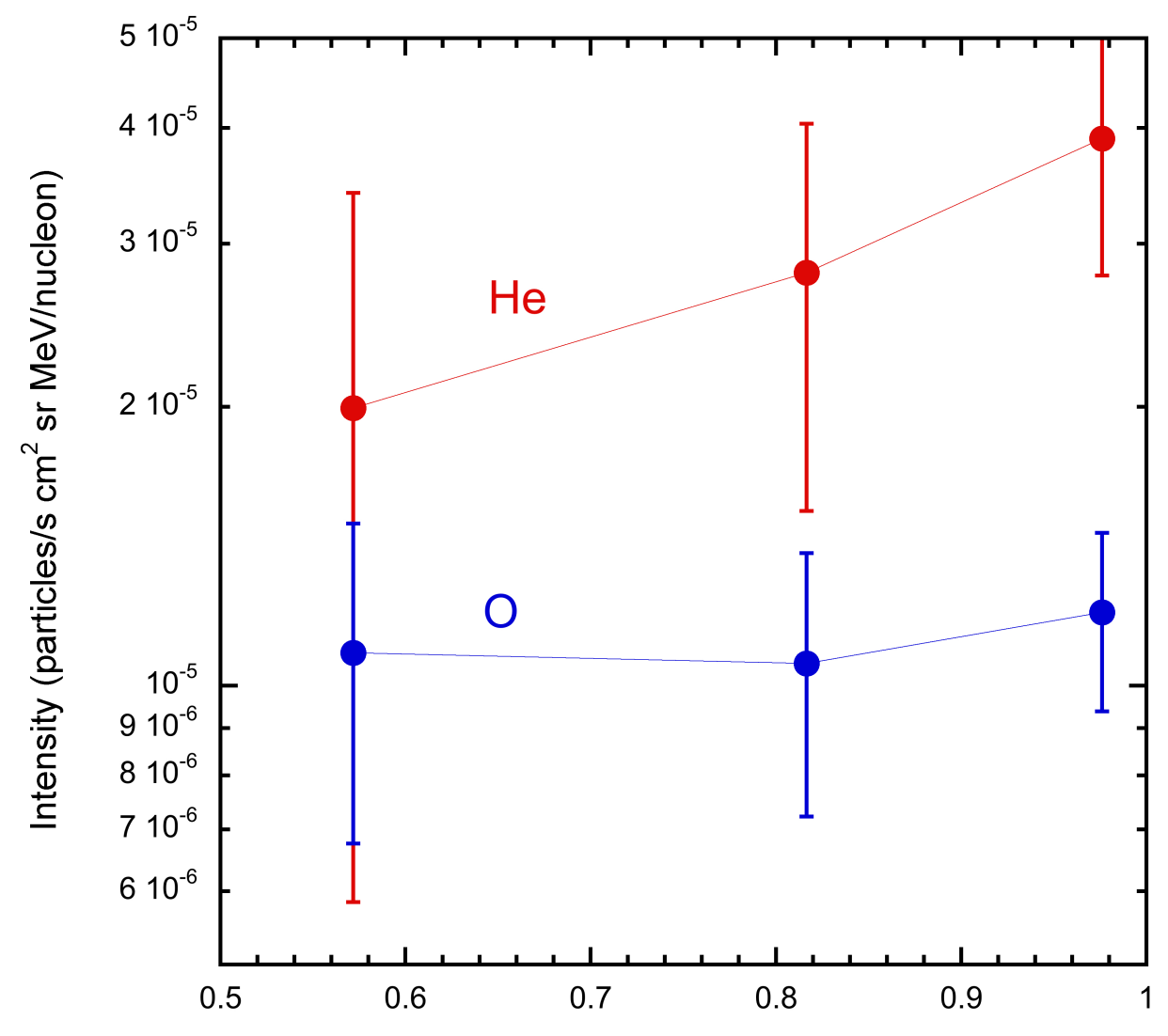

Heliocentric R au
Fig. A.1. Quiet-time interval $\mathrm{He}$ and $\mathrm{O}$ intensities observed near the peak of the low energy ACR spectra near 4.4 $\mathrm{MeV}_{\text {nucleon }}{ }^{-1}$ vs heliocentric radius. 


\section{Appendix B: Selection of super-quiet interval}

To select a super-quiet interval, we first selected intervals where suprathermal $\mathrm{H}$ and $\mathrm{He}$ intensities were noticeably lower than their values between intra-event peaks elsewhere. Figure B.1 shows three such areas in shaded yellow labeled (a)-(c) (e.g., compare days 130-138 with days 210-215). Summing over these three intervals yielded spectra similar to the low energy portions of Fig. 2, but at somewhat lower intensities. In particular the ${ }^{3} \mathrm{He} /{ }^{4} \mathrm{He}$ ratio was $(15 \pm 5) \%$, indicating an impulsive SEP source. Looking in more detail, we found that the arrival time of the ${ }^{3} \mathrm{He}$ showed that almost all of the counts arrived in a short period on day 164.9 in interval (c). The source of these particles might have been AR12765, which was in the Southern hemisphere at Carrington Longitude $113^{\circ}$ when viewed from Earth on day 165. Additionally, it was seen that during interval (b) the $\mathrm{H}$ and $\mathrm{He}$ intensities rose towards the end. Interval a did not show any such variations; rather, the intensities were low and did not show any obvious time variations. Interval (a) (2020 days 130.0-138.0) was therefore selected as the superquiet interval, and the other two were set aside. During the superquiet interval the Solar Orbiter separation angle from Earth was only about $9^{\circ}$, and so images from the Solar Dynamics Observatory (SDO) at L1 show the solar disc practically as viewed by Solar Orbiter. On NOAA reports for day 138, there were no plages or active regions, and the solar synoptic analysis was featureless except for small coronal holes. Solar Wind Ion Composition Spectrometer (SWICS) data from the ACE Science Center indicated slow $\left(\sim 280-350 \mathrm{~km} \mathrm{~s}^{-1}\right)$ solar wind speeds over the nine-day period (solar wind data from Solar Orbiter were not available). Thus, this extremely quiet period was associated with very low magnetic and solar wind activity. The interval was 192 hours long, and the average radial distance was $0.66 \mathrm{au}$.

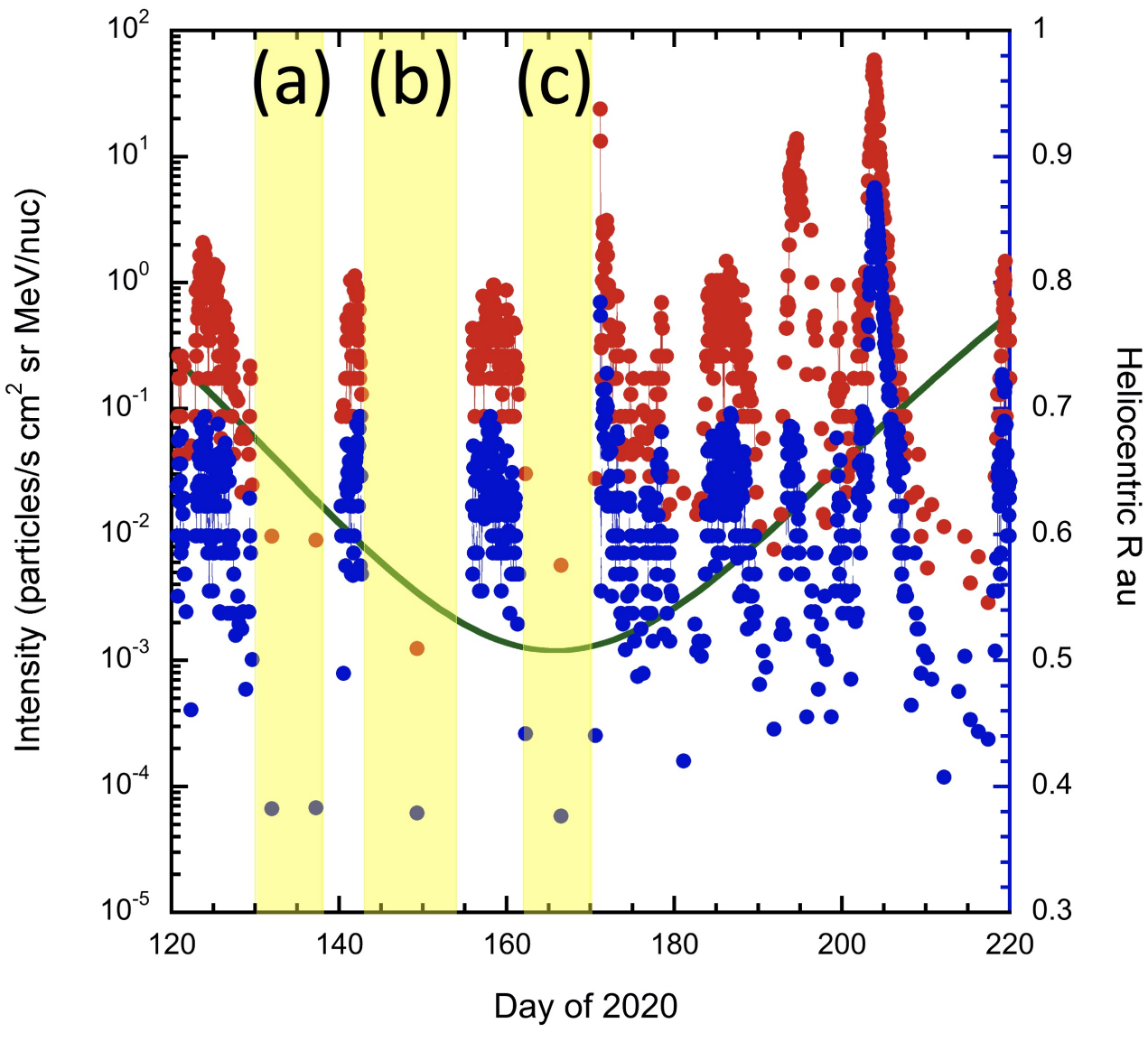

Fig. B.1. Intensities of $273 \mathrm{keV}$ nucleon $^{-1} \mathrm{H}$ (red filled circles) and $\mathrm{He}$ (blue), for mid-2020 showing heliocentric distance and yellow intervals $(a)$ (c) with low intensities. Large intensity increases in the figure are associated with CIRs and SEP events. The text describes residual time variations in intervals $(b)$ and $(c)$ that led them to be disqualified, leaving interval (a) as the super-quiet interval. 